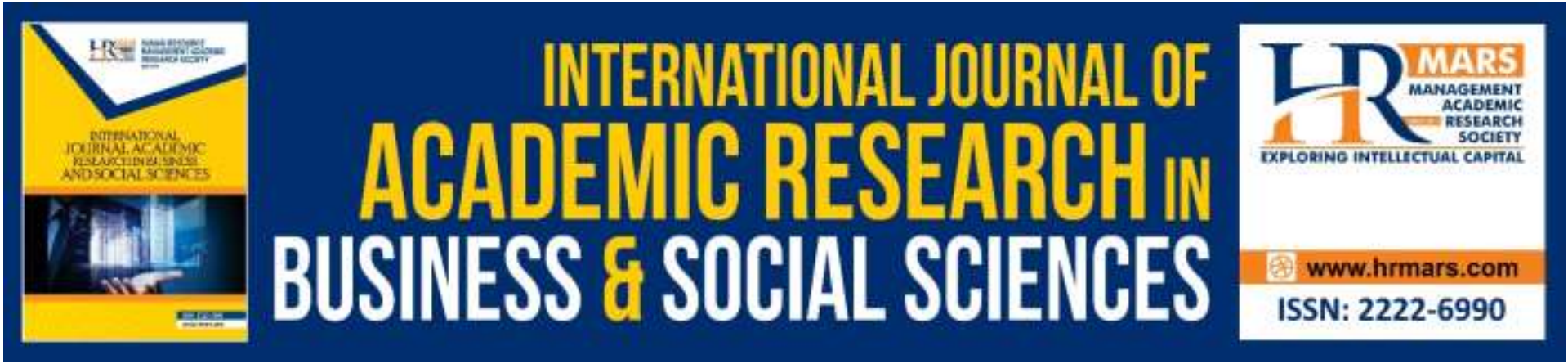

\title{
Contributor Factors for Psychological Well-Being: A Qualitative Study of Teachers' Perspective in Malaysia
}

Siaw Leng Chan, Mohamad Ibrani Shahrimin Adam Assim \& Fong Peng Lim

To Link this Article: http://dx.doi.org/10.6007/IJARBSS/v11-i17/11399 DOI:10.6007/IJARBSS/v11-i17/11399

Received: 12 August 2021, Revised: 14 September 2021, Accepted: 30 September 2021

Published Online: 19 October 2021

In-Text Citation: (Chan et al., 2021)

To Cite this Article: Chan, S. L., Assim, M. I. S. A., \& Lim, F. P. (2021). Contributor Factors for Psychological WellBeing: A Qualitative Study of Teachers' Perspective in Malaysia. International Journal of Academic Research in Business and Social Sciences, 11(17), 166-175.

Copyright: (C) 2021 The Author(s)

Published by Human Resource Management Academic Research Society (www.hrmars.com)

This article is published under the Creative Commons Attribution (CC BY 4.0) license. Anyone may reproduce, distribute, translate and create derivative works of this article (for both commercial and non-commercial purposes), subject to full attribution to the original publication and authors. The full terms of this license may be seen

at: http://creativecommons.org/licences/by/4.0/legalcode

Special Issue Title: Empowering Community and Beyond, iRandau, 2021, Pg. 166 - 175

Full Terms \& Conditions of access and use can be found at http://hrmars.com/index.php/pages/detail/publication-ethics 


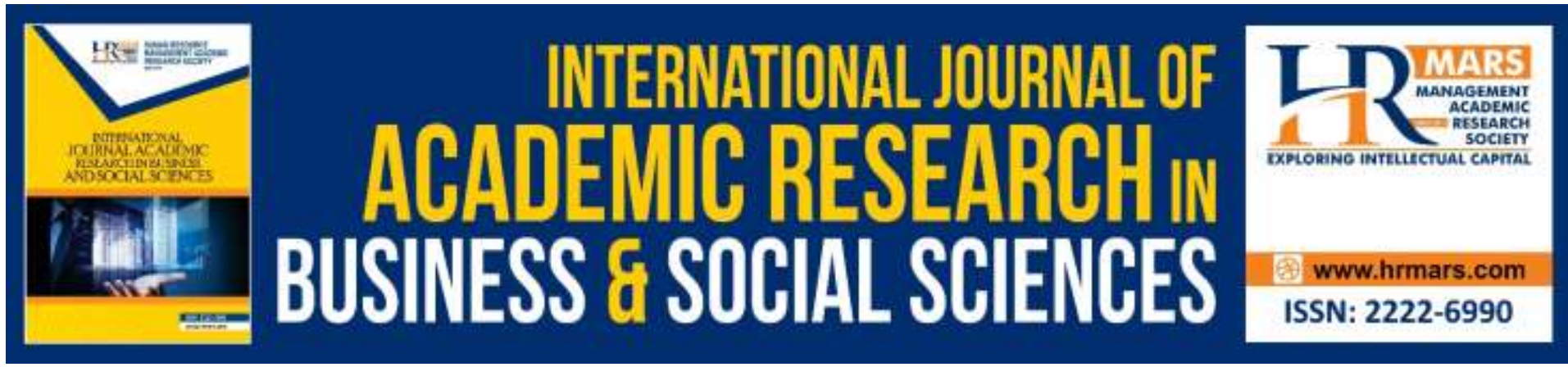

\title{
Contributor Factors for Psychological Well-Being: A Qualitative Study of Teachers' Perspective in Malaysia
}

\author{
Siaw Leng Chan ${ }^{1}$, Mohamad Ibrani Shahrimin Adam Assim¹ \& \\ Fong Peng Lim² \\ ${ }^{1}$ Department of Social Sciences and Management, Universiti Putra Malaysia Bintulu \\ Sarawak Campus, Sarawak, '2Department of Mathematics, Universiti Putra Malaysia, \\ Serdang, Selangor \\ Email: chansiawleng@upm.edu.my
}

\begin{abstract}
Universally, the educational systems are undergoing restructuring and transformations. The role of teachers has increased following this reformation and experiencing growing pressure to perform. Given that the nature of the teaching profession is both challenging and rewarding, it is vital to understand teachers' psychological well-being to support the mental health of this population. This study aimed to understand better the subjective perspectives regarding the contributor factors to psychological well-being among teachers. A consensual qualitative research approach was adopted in this study by collecting data through in-depth, semi-structured interviews. Interviews are used to understand the participants' narrative descriptions in-depth and uncover the meaning of their experiences, beliefs, and values. Seven Malaysian teachers were recruited from local school settings through purposive sampling. Data analysis involved an open-coding process in deriving themes from the participants' responses. Findings revealed five themes that indicate contributor factors for psychological well-being: positive attitudes, spiritual convictions, family and friends' support, personal growth, and personal responsibility. The practical implications of teachers' psychological well-being, mental health and psychotherapy practice, and future research were also discussed in this work.
\end{abstract}

Keywords: Contributor Factor, Perspective, Psychological Well-Being, Qualitative Study, Teacher

\section{Introduction}

Undoubtedly, teachers in Malaysia are the most devoted in the world to ensure their students acing academically. For instance, the Global Education Census Report Malaysia (2018) highlighted that those Malaysian teachers documented the highest percentage of teachers who dedicated additional time to ensure students understand the importance of excelling in their assessments. Malaysian teachers are very centred on assisting their students in attaining good grades and achieving their career ambitions. The teaching profession is conceded as 
both challenging and satisfying as they are to be one of the professionals essential to construct and educate future leaders.

Given that the educational systems are undergoing restructuring and transformations globally, the role of teachers has expanded following this reformation and thus far, experiencing growing pressure to perform. Consequently, teacher psychological well-being is vital to the supportive teacher-student relationship, teaching-learning process, and learning effectiveness. As a developing country, Malaysia has retained more prominence on the population's mental health, including teachers, whereby grasping, and practising psychological well-being is vital for professional motivation (Jeon et al., 2019). Subsequently, psychological well-being has emerged as a healthy and positive attitude in enhancing teachers' psychological well-being. However, these essential concepts have not been given much attention in Malaysia, particularly from the teachers' perspectives.

Lack of psychological well-being amongst teachers is frequently associated with depressive and psychosomatic symptoms, alcohol consumption, and burnout (Schonfeld, Bianchi \& Luehring-Jones, 2017). Therefore, reviewing their psychological well-being can help teachers improve their personal growth and strengthen the capability to utilise the self competently in the teaching-learning relationship. Additionally, past research indicated that well-being mediation plays a substantial role in enhancing teachers' teaching value, selfcompassion, and feelings of satisfaction, thus cultivating students' self-reported temperaments that heighten classroom excitement parameter, concentration, attention, and learning (Bradley et al., 2018).

Previous studies revealed several impediments and mediators in terms of perceived contributor factors affecting teachers' psychological well-being. For instance, contributor factors like teachers' resilience, workplace societal support, opportunity to get promoted and develop self-worth, and accommodating work settings were linked positively with their psychological well-being (Brouskeli, Kaltsi, \& Loumakou, 2018; Jeon, Buettner, \& Grant, 2018; Malik, Björkqvist, \& Österman, 2017). On the other hand, factors such as office bullying, stringent authority's bureaucratic approach, inadequate facilities at school, negative attitudes of colleagues, the poor reputation of the profession, and teachers' negative experiences reduced teachers' psychological well-being holistically (Çimen, \& Ozgan, 2018; Malik et al., 2017).

The notion of psychological well-being is encompassed of six distinctive subdimensions, namely purpose in life, personal growth, autonomy, self-acceptance, environmental mastery, and positive relationships with others (Ryff, 1989). These dimensions contribute to individual personal development, which enhances their life satisfaction (Ryff, 1989).

This study carried out a qualitative study that aimed to explore teachers' experiences in Malaysia who engaged in teaching and learning processes, including their perspectives on psychological well-being and perceptions on contributory factors in enhancing their psychological well-being. Hence, the central research question was: What are the perceived contributors to psychological well-being among schoolteachers in Malaysia? This research will focus on the facilitating factors of the educators' psychological well-being from their unique perspectives.

\section{Methods}

The current research study utilised a consensual qualitative research approach to provide an in-depth understanding of subjective perspectives regarding the perceived contributor 
factors to psychological well-being among teachers. A consensual qualitative study (CQR) design was applied in the current study, using in-depth and partially structured interviews to collect and support audio recordings and interview transcripts (Hill and Knox, 2021).

This CQR method is often used as a qualitative research method in counselling psychology research (Hill and Knox, 2021). It is designed and used by Hill (2012) and Anderson et al. (2014), who propose semi-structured interviews. Creswell (2012) highlighted that the basic idea behind this specific research method is "multiple researchers, reaching consensus as a team, and a systematic methodological approach to identifying the representation of study data across cases". Hill, Thompson, and Williams (1997) have described the CQR process as a process involving three general steps, namely: a) the response to open-ended questions through questionnaires or interviews for each case is divided into domains (or topic areas); $b$ ) core ideas (abstract or summary) are constructed for all materials in each domain for each case; c) cross-analysis involving category expansion to illustrate consistency in core ideas in domains across cases.

\section{Participants}

This study recruited seven (7) Malaysian teachers from local school settings through purposive sampling. Study participants were teachers with at least three years of work experience (see Table 1). In the present study, seven teachers were interviewed by the researcher, with each interview lasted 40 to 60 minutes. Seven teachers (three male and four female), aged between 25 and 47, participated in this study. In terms of years of service, two (2) teachers served three years (code P1 and P6), one (1) served eight years (code P2), one (1) served ten years (code P3), one (1) served eighteen years (code P4), one (1) served eleven years (code P5), and one (1) served fourteen years (code P7). In terms of ethnicity, the study participants consisted of two (2) Malays, two (2) Chinese, two (2) indigenous, and one (1) Indian.

Table 1: Demographic Summary of Participants

\begin{tabular}{lllll}
\hline Participant [P] & Gender & Age & Race & Year(s) of Service \\
\hline P1 & Female & 27 & Chinese & 3 \\
P2 & Female & 32 & Chinese & 8 \\
P3 & Male & 35 & Malay & 10 \\
P4 & Male & 47 & Indigeneous & 18 \\
P5 & Female & 37 & Malay & 11 \\
P6 & Male & 28 & Indigenous & 3 \\
P7 & Female & 42 & Indian & 14 \\
\hline
\end{tabular}

\section{Data Collection}

For this study, informed consent forms were acquired from all seven research participants. Data was gathered through seven interview sessions (one session per participant). The 
procedure by Schuman (1982) was used as the primary source in preparing the interview protocol (Creswell, 2012). This study used semi-structured interviews to allow various roles and experiences to emerge through the following questions: (1) What are your experiences working as a teacher? (2) What are the challenges faced during the teaching-learning process? (3) What are the perceived contributors to your psychological well-being? For confidentiality and to protect the anonymity of study participants, a code was used [Participant (P)], and each participant agreed on it. The researcher conducted online and one-to-one interviews in either Malay or English (at the participants' option), and each interview takes about 40 to 60 minutes.

\section{Data Analysis}

The findings of the study data were analysed using a thematic approach (Braun \& Clarke, 2006) that complied with three stages of the coding process, namely: open coding, axial coding, and selective coding. Researchers utilised an open-coding method to identify themes that arose through raw data obtained from study participants. In qualitative research, it is vital to consolidate the results coded by the researcher to reduce bias and ensure transparency of the findings (Guion, 2002). Therefore, member checking and external evaluator are used to providing the trustworthiness of the whole study.

\section{Results}

Findings revealed five themes that indicate contributory factors for psychological well-being: positive attitudes, spiritual convictions, family and friends' support, personal growth, and personal responsibility (see Figure 1). Remarkably, each theme was interrelated in contributing to psychological well-being among teachers. From this study, the perceived facilitators of psychological well-being involved dynamic developments. 


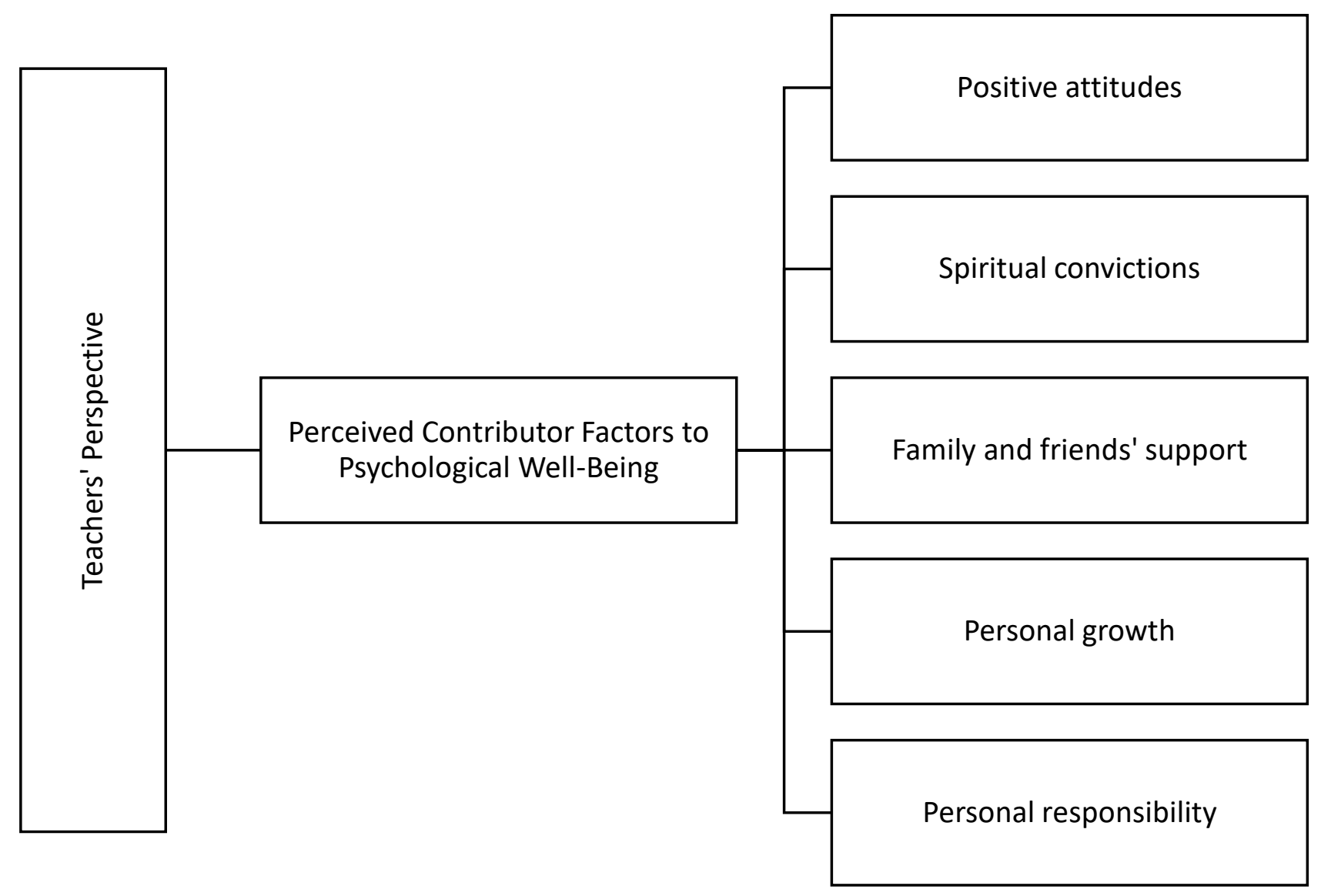

Figure 1: Summary of emerging themes of perceived contributor factors to psychological wellbeing

\section{Theme 1: Positive Attitudes}

One of the most expressed contributing factors shared by the participants was positive attitudes in psychological well-being. The participants perceived thinking, feeling, and acting positively on challenges as crucial in increasing an individual's psychological well-being.

'It's not easy to handle all the tasks at the same time. My positive attitude when facing any problems helps a lot.' (P1)

'Positive thinking. I always tell myself to look at things positively and that everything has a solution.' (P3)

'I believe that being genuine, fair and free-spoken in making every decision (always) helps me a lot as a teacher. Thus, my positive attitude helps me (a lot) to encourage myself to explore more good things in life.' (P4)

'Good emotion and good communication. I believe that will help me.' (P7)

\section{Theme 2: Spiritual Convictions}

Spiritual convictions or beliefs played as vital energy to their psychological well-being, which had presented by participants. Participants revealed that they would depend on spiritual trust when things get more challenging to handle emotionally and mentally. 
'More empathy less apathy. Accept that as humans, we make mistakes, humans may die anytime, and even robots may not function. Get back to God.' (P2)

'In fact, I am a very spiritual and punctual person.' (P4)

'Belief in Allah, everything will go under control.' (P5)

\section{Theme 3: Family and Friends' Support}

Participants believed that through supportive relationships with family and friends (including colleagues), they experienced great contentment when they aided, encouraged, tried to understand, listen to, and motivate them. Essentially, the core in such supportive relationships made the participants feel the care, love, and presence (not alone).

'Moral support, especially from family members and friends, are significant to me. Whenever I feel stress, I need to talk about it with my friends. They always listen to me and gave me moral support.' (P2)

'Love, compassion and tolerance from all sides to support each other.' (P3)

'Moral support from my family helps me the most.' (P5)

'All teachers and staffs need to consider and support each other in making activities successful.' (P7)

\section{Theme 4: Personal Growth}

The participants shared about the need to focus on personal development and improvement. Some participants discovered external strengths in terms of skills that have helped them be strong and improve self-confidence.

'Improvement of my teaching skill. Many things changed, and we have to keep up with the transformation, especially with students.' (P1)

'Try something new to improve self-confidence in teaching.' (P4)

'The openness of sharing among teachers help in improving teaching methods and students' well-being.' (P6)

\section{Theme 5: Personal Responsibility}

The participants shared the importance of appreciating the need to love themselves first if they wish for changes. Without acknowledging their responsibility, they could face difficulties to break through their emotional barriers to relieve themselves and seek out help from others.

'Self-discipline. Stated roadmaps to achieve goals. Stick to one thing first, and you can observe how we can do things. It is not for the short term but the long-term process.' (P2)

'Self-reward after achieving or completing specific tasks. It is for the appreciation on achievement and hard work done.' (P3) 
'Better time management. When working, then work. When relaxing time, then rest and love ourselves more. It's our choice to balance up our work and life.' (P5)

\section{Discussions}

The findings of our study reveal a description of the teachers' perspective of contributory factors to psychological well-being. A total of five contributory factors to psychological wellbeing have been discovered: (1) positive attitudes, (2) spiritual convictions, (3) family and friends' support, (4) personal growth, and (5) personal responsibility (see Figure 1). When the participants shared their experiences and perspectives, there were unique interconnections. For instance, one facilitator factor relates to another factor (e.g., positive attitudes and personal growth).

In this study, positive attitudes and personal growth are depicted as essential factors contributing to their psychological well-being (Bradley et al., 2018; Çimen \& Ozgan, 2018). Essentially, looking at the positive sides during challenging situations and acknowledging personal development will help provide a holistic understanding of their strengths and weaknesses more healthily.

Given the heightened responsibility of teachers' roles, the teaching profession can be rewarding and stressful simultaneously (Jeon, Kwon, Walsh, et al., 2019). The present study indicated that receiving supports from family and friends and as well as spiritual convictions have played an essential role in teachers' psychological well-being (Brouskeli, Kaltsi, \& Loumakou, 2018; Jeon, Buettner, \& Grant, 2018; Malik, Björkqvist, \& Österman, 2017). The fundamental supportive relationships made the participants felt they were cared for, loved, and motivated. Besides, spiritual convictions are portrayed as a core in renewing their belief systems throughout the self-care process.

The participants believed that personal responsibility also played a vital aspect in their psychological well-being (Çimen, \& Ozgan, 2018; Jeon, Kwon, Walsh, et al., 2019; Malik et al., 2017). The desire to be responsible to themselves in facing difficulties was perceived to help them break through their emotional barriers and seek out help from others whenever they break down or feel devastated.

The current study contributes to the knowledge and practice of psychological wellbeing in the Malaysian context, particularly among teachers. By adding real-life experiences to the stream of research focusing on teachers' psychological well-being, mental health and psychotherapy practitioners can enhance their clients' psychological health.

Moreover, the study's findings on teachers' perspectives on contributory factors to psychological well-being provide practical, evidence-based information on professional training. Subsequently, this development can improve teachers' psychological well-being in the long term and holistically.

The current findings may help alleviate the issue of inadequate emphasis in the Malaysian education system in training teachers about psychological well-being by incorporating the concept of psychological well-being into its syllabus and programs.

Furthermore, this study expands the study of psychological well-being in Malaysia and extends existing knowledge of psychological well-being from a non-Western perspective. Additionally, the study's findings may indicate other issues, such as the importance of selfcare among teachers. Hence, school administrators can create a culture of self-care in local schools by promoting the importance of psychological well-being. This supportive environment may help make healthy behaviours in teachers at the beginning of their career, enhancing wellness and decreasing the risk of psychological impairment in the future. 


\section{Limitations}

One limitation is that the study only centres on Malaysian secondary school teachers' experiences, making it difficult to extend to educators in other areas. Given the small sample size, future research is essential to ascertain whether the present findings are consistent with another sample of educators in different educational institutions. The study also aims at the general context of psychological well-being. Future research ought to examine specific subdimension of psychological well-being among different cultures.

\section{Conclusion}

In conclusion, all five themes that emerged from teachers' perspectives of contributory factors to psychological well-being have facilitated the participants to manage their work and life. As such, in the context of a swiftly shifting and challenging teaching profession, a constant discussion of psychological well-being for teachers appears necessary and timely. Each facilitator factor is interconnected for participants to improve their psychological well-being. It is essential to be mindful of and understand the aspect of each theme as this provides more objective and holistic ideas in understanding teachers' psychological well-being in ensuring the continuation of this noble profession.

\section{Acknowledgement}

This research was supported in part by Universiti Putra Malaysia Grant (Geran Putra-Inisiatif Putra Muda, ID: GP-IPM/2020/9684700). We are thankful to all participants for their willingness to be part of the study. Special thank you to the Ministry of Education, Malaysia, for the permission to collect data in respective local schools.

\section{References}

Anderson, C. A., Leahy, M. J., DelValle, R., Sherman, S., \& Tansey, T. N. (2014). Methodological application of multiple case study design using modified consensual qualitative research (CQR) analysis to identify best practices and organisational factors in the public rehabilitation programme. Journal of Vocational Rehabilitation, 41, pp.87-98.

Bradley, C., Cordaro, D. T., Zhu, F., Vildostegui, M., Han, R. J., Brackett, M., \& Jones, J. (2018). Supporting improvements in classroom climate for students and teachers with the four pillars of well-being curriculum. Translational Issues in Psychological Science, 4(3), 245-264. DOI: 10.1037/tps0000162

Braun, V., Clarke, V. (2006). Using thematic analysis in psychology. Qualitative Research in Psychology, 3, 77-101. doi:10.1191/1478088706qp063oa

Brouskeli, V., Kaltsi, V., \& Loumakou, M. (2018). Resilience and occupational well-being of secondary education teachers in Greece. Issues in Educational Research, 28(1), 43-60.

Çimen, I., \& Ozgan, H. (2018). Contributing and damaging factors related to the psychological capital of teachers: A qualitative analysis. Issues in Educational Research, 28(2), 308.

Creswell, J. W. (2012). Qualitative inquiry and research design: Choosing among five approaches (3rd ed.). Thousand Oaks, CA: SAGE (n.a.). Global Education Census Report 2018. Cambridge Assessment International Education Shaftesbury Road, Cambridge CB2 8EA, United Kingdom, 2018. Print.

Guion, R. M. (2002). Validity and reliability. In S. G. Rogelberg (Ed.), Blackwell handbooks of research methods in psychology. Handbook of research methods in industrial and organisational psychology (p. 57-76). Blackwell Publishing. 
Hill, C. E. (ed.) (2012). Consensual qualitative research: A practical resource for investigating social science phenomena. Washington DC: American Psychological Association.

Hill, C. E., \& Knox, S. (2021). Essentials of Consensual Qualitative Research. Washington DC: American Psychological Association.

Hill, C. E., Thompson, B. J., \& Williams, E. N. (1997). A guide to conducting consensual qualitative research. The Counseling Psychologist, 25(4), pp.517-572.

Jeon, L., Buettner, C. K., \& Grant, A. A. (2018). Early childhood teachers' psychological wellbeing: Exploring potential predictors of depression, stress, and emotional exhaustion. Early education and development, 29(1), 53-69. DOI: 10.1080/10409289.2017.1341806

Jeon, H. J., Kwon, K. A., Walsh, B., Burnham, M. M., \& Choi, Y. J. (2019). Relations of early childhood education teachers' depressive symptoms, job-related stress, and professional motivation to beliefs about children and teaching practices. Early education and development, 30(1), 131-144. DOI: 10.1080/10409289.2018.1539822

Malik, N. A. A., Björkqvist, K., \& Österman, K. (2017). Factors associated with occupational stress among university teachers in Pakistan and Finland. Journal of Educational, Health, and Community Psychology, 6(2), 1-14.

Ryff, C. D. (1989). Happiness is everything, or is it? Explorations on the meaning of psychological well-being. Journal of Personality and Social Psychology, 57(6), 10691081. DOI:10.1037/0022-3514.57.6.1069

Schonfeld, I. S., Bianchi, R., \& Luehring-Jones, P. (2017). Consequences of job stress for the psychological well-being of teachers.

Schuman, D. (1982). Policy analysis, education, and everyday life. Lexington, MA: Heath. 